\title{
LOS CIRCULOS VIRTUOSOS, LA PLATAFORMA PARA TRANSMITIR VALOR. SU APORTE EN ESCENARIOS DE INCERTIDUMBRE Y AMENAZA
}

\section{AMIGO, Adriana Célide}

\section{RESUMEN:}

El presente artículo analiza la importancia de la construcción de anclas positivas inspiradoras y facilitadoras, en el escenario empresarial, cuando toda vez que la adversidad, la incertidumbre y la amenaza del futuro se presenta, se necesite replantear la mirada hacia el futuro para que todos caminen en la misma dirección. Pero los círculos virtuosos, que podrían también considerarse usinas de desarrollo interno, no representan el soporte ante la adversidad, también lo son cuando las estrategias son exitosas, puesto que permitirán potenciarlas y preservarlas.

PALABRAS CLAVE: Construcción; Círculo; Virtuoso; Soporte; Estrategias

\begin{abstract}
:
This article analyzes the importance of the construction of inspiring and facilitating positive anchors, in the business scenario, when whenever adversity, uncertainty and the threat of the future appear, it is necessary to rethink the look towards the future so that everyone can walk in the same direction. But virtuous circles, which could also be considered internal development plants, do not represent support in the face of adversity, they are also so when strategies are successful, since they will allow them to be strengthened and preserved.
\end{abstract}

KEYWORDS: Construction; Circles; Virtuous; Support; Strategies

\section{INTRODUCCIÓN}

Las empresas que desarrollan su estrategia sobre la base de la correcta definición del valor a brindar para una categoría de clientes que perciben, identifican, diferencian y eligen a ese valor, habrán sustentado su estrategia en el proceso de desarrollar ventajas competitivas. (Amigo, 2012; 2017)

Las empresas que permanecen en ese mercado, con carácter de continuidad están conviviendo con la trascendencia. Las empresas que trascienden compiten. Las empresas que compiten están caminando hacia el futuro.

Pensar en los objetivos, es intentar caminar hacia el futuro. Pero ¿Cómo se vislumbran? Se vislumbran en el presente, teniendo en cuenta que dichas declaraciones de principios o de intenciones deben formularse, sabiendo que con ellos comienza el proceso de la autoevaluación de la estrategia, y asegurando que sin ellos el crecimiento no existe. Los 
objetivos, establecen el quiebre entre la realidad y lo esperado. Debemos plantearnos si las empresas en escenarios volátiles, cambiantes, inesperados, establecen la cultura de los objetivos. Quizás la respuesta sea negativa, pues el devenir de la realidad, superponga los horizontes de tiempo y las acciones consecuentes. $Y$ el proceso de fijación de objetivos, es la base para empezar a formular los círculos virtuosos, en los que todas las personas se sienten incluidas e inspiradas. Más que difícil formular objetivos, lo es mucho más el tiempo en que las personas decidan comenzar a gestarlos para más luego cumplirlos. ¿Qué pasa si cuando miramos hacia el futuro, nutrimos nuestra mirada de valor?, con:

1. Requerimiento

2. Oferta genuina

3. Demanda sensible al beneficio o concepto

4. Voluntad de pagar por el valor

5. Precio equitativo para ambas partes

6. Continuidad de gestión del valor

Quizás entonces, los objetivos comiencen a cobrar una vida más comprometida, con el futuro, con la continuidad, con la trascendencia, con la Visión y con la permanencia de la Misión.

\section{EL VALOR DE LA CREATIVIDAD}

El proceso de la formulación de objetivos es un proceso comprometido, virtuoso, corporativo y hasta desestabilizador, pues en primer lugar deben formularse en un proceso de negociación interna, en el que se prioriza el equilibrio de la organización, como Simon (1980) expresa en su obra "El Comportamiento Administrativo".

La clave de la participación de los individuos en la organización, estriba en (...) el grupo organizado como como sistema de equilibrio. Los individuos están dispuestos a aceptar su condición de miembros de la organización, cuando su actividad dentro de ella contribuya, directa o indirectamente, a sus propios fines personales. Esa contribución es directa si las finalidades de la organización tienen para el individuo un valor personal directo (Simon, 1980, p. 105).

Sería muy simple, listar las áreas comprometidas con el futuro, reclamarles quantums de objetivos para dentro de seis meses y luego medir el desempeño en función de tales estándares.

Pero vislumbrar el futuro con valor, implica comprometerse con una mejor historia en donde los protagonistas sean la sociedad, el sector industrial, los proveedores, el cliente interno, el cliente externo y la propia empresa.

John V. Buckley (1999), al hablar del desafío de Reuters, dice que para que Reuters logre un crecimiento continuo, deberá asegurar que las funciones de desarrollo proporcionen lo 
que los tecnólogos consideren que pueden dar y lo que los comercializadores de la compañía piensen que desea al mercado. (Amigo, 2012; 2017) Y si Reuters, pretende seguir en pos del crecimiento con base en una trayectoria marcada por un éxito tras otro, va a requerirse un trabajo más próximo entre estos dos grupos a medida que los grupos comprometidos en el crecimiento se proyecten hacia el futuro

No todas las combinaciones generan la mejor propuesta de valor, ni tampoco son igualmente transcendentes para sostener y desarrollar el perfil competitivo de la empresa, cuando además desde el punto de vista estratégico esa combinación tiene que ser tal que permitan transformarse en capacidades distintivas, o sea aquellas capacidades que reúnan las siguientes característica: escasas, de difícil imitación, sin substitutos, valoradas por el mercado y de difícil venta (Amigo, 2009; 2012; 2017).

El solo hecho de pensar en una propuesta de valor para un mercado, crea libertad para la estrategia. Y cuando se inicia este proceso de pensamiento, se están gestando ideas ¿Las ideas en qué se sustentan? Las ideas surgen de las ideas. ¿Cuáles?

Las ideas que se sustentaron en la investigación de las distintas categorías de comportamientos, los comportamientos de los grupos que aglutinan valor, los llamados GAVs (Amigo, 209; 2012; 2017): internos, externos, esporádicos, continuos, estratégicos, operativos.

El grupo aglutinador de valor es el principal sustentador de las ideas. Pero ¿qué pasa si la empresa no descubre sus distintos GAVS? Quizás pierda, la principal fuente de inspiración de sus ideas y de la continuidad de su estrategia.

Según Buckley (1999), la mayor parte de las organizaciones exitosas, en cualquier industria donde operen, cuentan con un proceso institucionalizado de estímulo y manejo de la creatividad y la innovación.

Pedir a los miembros del staff, provenientes de diferentes funciones para que se sienten alrededor de una mesa y sean creativos, seguramente producirá una lluvia de nuevas ideas, pero ¿Cuál será el resultado? Se hace necesario, construir una estructura para el desarrollo de ideas innovadoras, en las que se puedan obtener y evaluar todas las buenas ideas innovadoras y en las que se puedan tomar las incrementales como las devastadoras. No se me hace fácil pensar en las devastadoras si las devastadoras, destruyen un mercado en desarrollo. ¿Qué pasa cuando el mercado comienza a crecer, se empieza a poblar de oferentes innovadores y clientes imitadores y la tecnología irrumpe, para romper ese equilibrio incremental? Eso, es ¿valor, continuidad o desvalor? ¿Qué paso con la creatividad en los últimos dos años, ante la adversidad inesperada de una pandemia mundial?

Desde el inicio de la crisis sanitaria, social y económica en la que nos encontramos inmersos actualmente, diversas marcas están generando movimientos de respuesta de los 
más originales para combatir al COVID-19. A este hecho, hay que añadir que el distanciamiento provocado por la Pandemia en las organizaciones ha demostrado que la cultura corporativa y las relaciones entre compañeros y gerentes son estrategias clave que se deben potenciar. Los departamentos de comunicación hasta la fecha no disponen de herramientas con capacidad analítica que les permita medir directamente el impacto en su organización de los intangibles. La crisis del COVID-19 ha evidenciado la importancia de calcular el valor de marca, la reputación, la imagen, la experiencia de compra y las relaciones que establece. $Y$ no únicamente lo que se ha vendido, que además ha sido muy poco en medio de un escenario profundamente recesivo. Es aquí donde cobran gran importancia las decisiones que se van a tomar para posicionar la marca adecuadamente cuando el virus haya sido erradicado.

El manejo de la creatividad, es la etapa en la cual se desarrollan y refinan progresivamente las ideas creativas. La planeación para el crecimiento exige una transformación de la generación de ideas hacia la planeación e implementación de proyectos. (Amigo, 2012; 2017)

Un ejemplo de la creatividad al servicio del valor, inspirado en la pandemia decretada por la OMS, son algunas de las siguientes campañas de marcas que reformularon sus logos en algunos casos para adecuarlos a sus objetivos de comunicación, para transmitir e inspirar conciencia.

\section{LAS CAMPAÑAS DE LAS MARCAS, AL SERVICIO DE LOS CíRCULOS VIRTUOSOS}

Un ejemplo de creatividad al servicio del valor, lo representaron muchas empresas que ante la transversalidad de la pandemia, decidieron crear conciencia a partir de sus políticas de branding, generando inspiración para la conciencia colectiva.

\section{LA CAMPAÑA DE AUDI}

Audi, desarrolló una campaña destinada a concientizar a la población sobre la necesidad de mantener las distancias entre personas "para estar más unidos" frente la lucha contra el coronavirus. La marca de coches en su forma para mostrar solidaridad y empatía con el distanciamiento social, decidió separar los círculos tradicionales que componen el logotipo de la marca. 
GRAFICO 1. La campaña de Audi. Fuente (Atrivity, 2020)

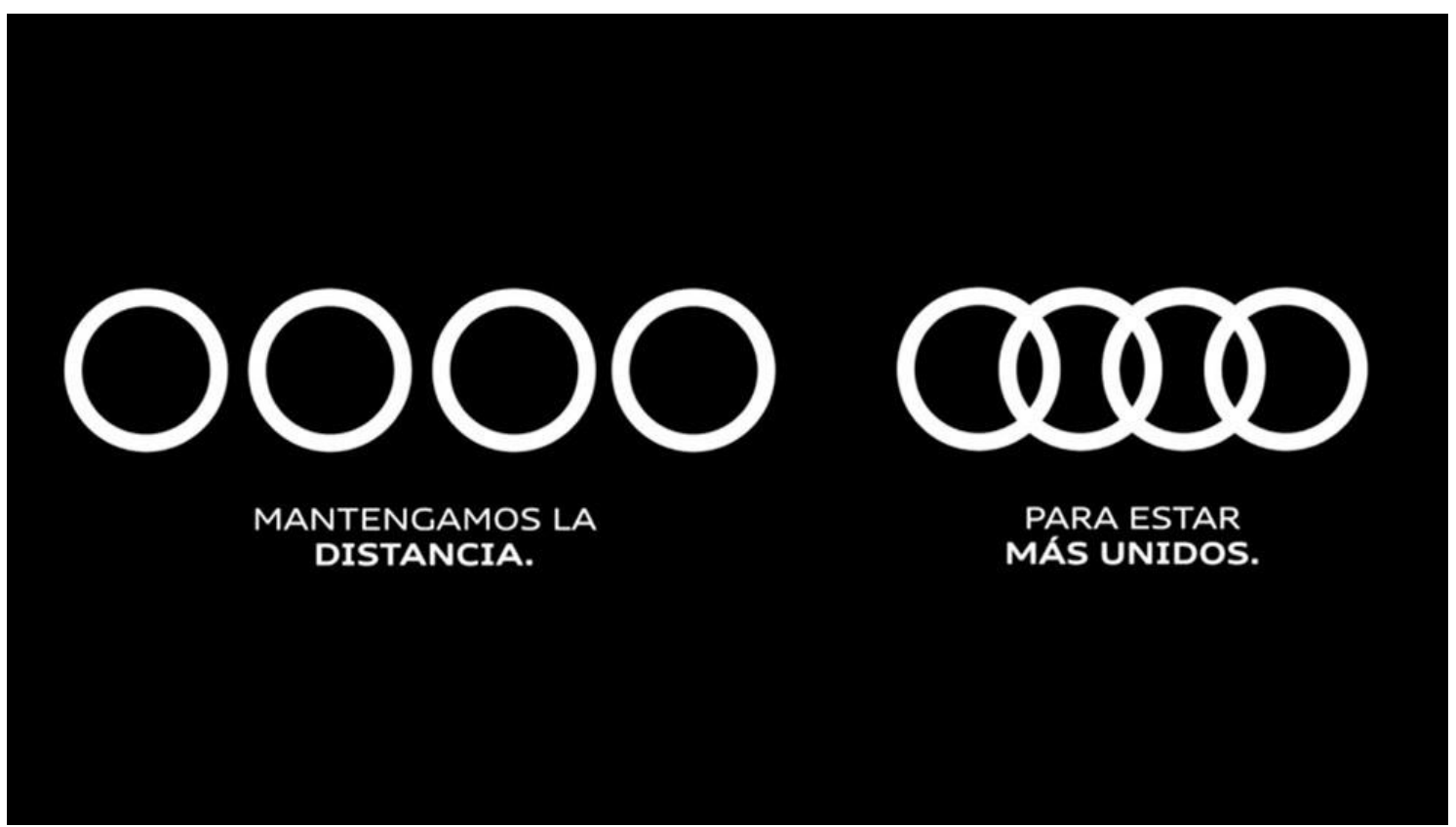

\section{LA CAMPAÑA DE NIKE}

Nike, utilizó como referencia el sueño de cualquier amante del deporte como es jugar en grandes estadios con millones de espectadores y ser decisivos para conquistar un título. Ese sueño Nike lo hace realidad: es el momento en el que todos estamos jugando el partido más importante de nuestras vidas y lo tenemos que jugar quedándonos en casa. El eslogan utilizado fue "play inside, play for the world".

GRÁFICO 2. La campaña de Nike. Fuente. (Atrivity, 2020)

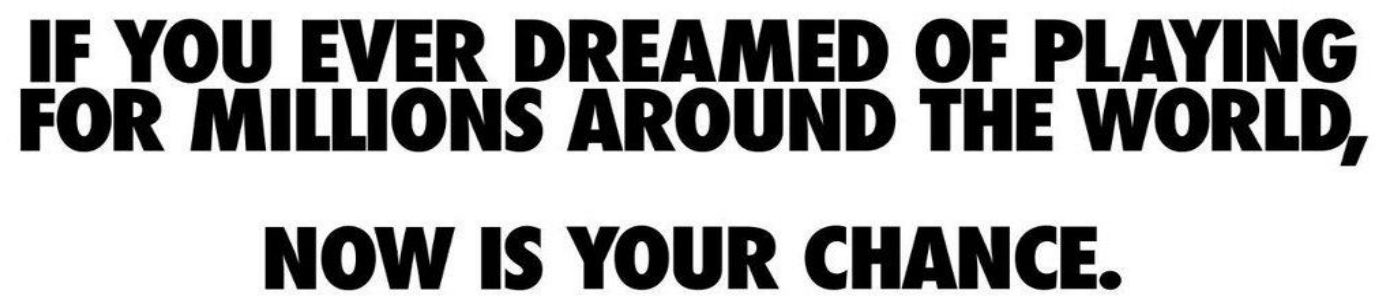

Play inside, play for the world.

\section{LA CAMPAÑA DE MERCEDES BENZ}

Mercedes Benz es muy rigurosa a la hora de seguir las recomendaciones sanitarias, en especial la de "quedarse en casa", "lavarse las manos" y todas las medidas necesarias para "evitar más contagios". Para ello, ha utilizado en redes sociales la imagen de varios de sus modelos $4 \times 4$ cruzando zonas arriesgadas para ilustrar el lavado de manos. 
GRAFICO 3. La campaña de Mercedes Benz. Fuente (Atrivity, 2020)

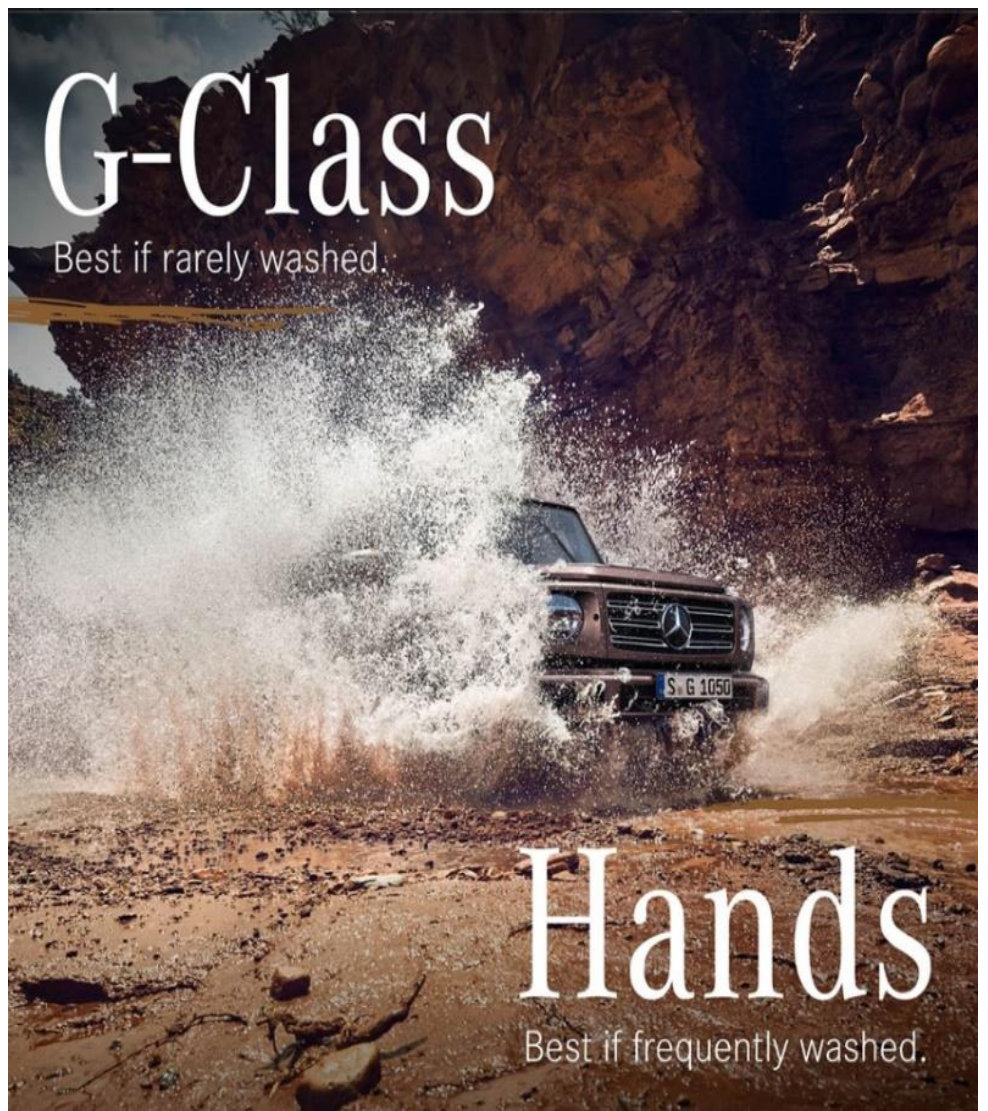

\section{ESTRATEGIAS DE RESPONSABILIDAD SOCIAL CORPORATIVA}

Este tipo de campañas, tienen por objetivo, desarrollar, comunicar y posicionar las acciones que realizan las empresas sin ánimo de lucro como colaboraciones con fundaciones, disminución de la contaminación o donaciones. En este caso organizaciones como el Banco Santander, Ikea e Inditex, son protagonistas por sus cuantiosas donaciones de material médico, mascarillas, cuentos para niños y poner a la disposición de su potencial logístico para almacenarlo y distribuirlo. Estas acciones han sido de gran importancia por la escasez de este tipo de recursos en los centros hospitalarios.

\section{LA ESTRATEGIA DE RESPONSABILIDAD SOCIAL CORPORATIVA DE Banco Santander.}

La entidad bancaria por su parte donó un millón de mascarillas, 2.000 camas de hospital, respiradores y material sanitario. En este contexto de emergencia es una de las compañías que ha ofrecido sus recursos para combatir el grave impacto provocado por la enfermedad. El Santander se encargó de realizar los envíos a toda la península del material con el hashtag \#EsteVirusLoParamosUnidos. También cabe destacar que abrió una línea de 
financiación para PyMEs y autónomos de 20.000 millones para asegurar la liquidez al corto plazo.

GRÁFICO 4. La estrategia de RSC de Banco Santander, Fuente (Atrivity, 2020)

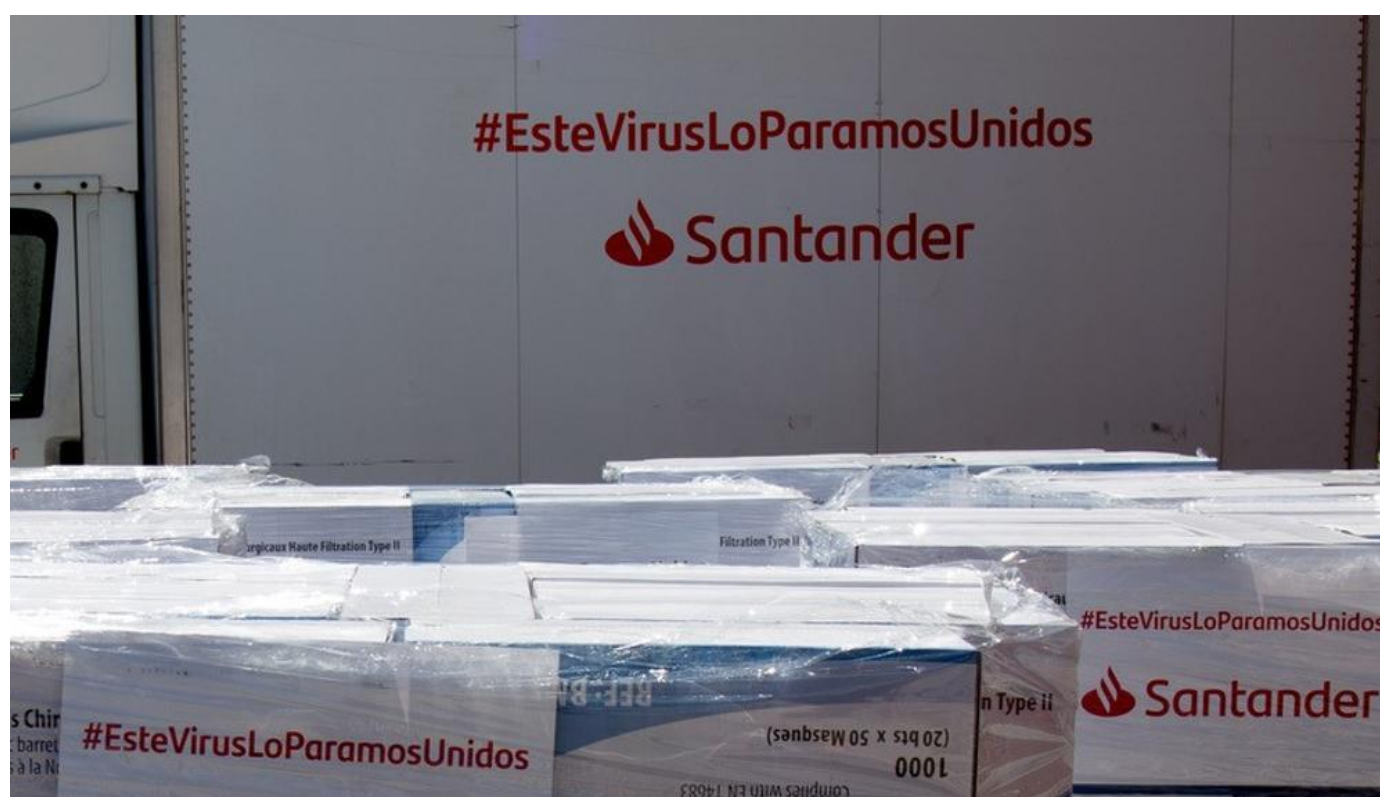

\title{
LA ESTRATEGIA DE RESPONSABILIDAD SOCIAL CORPORATIVA DE Ikea.
}

Una empresa siempre enfocada a mejorar y organizar el interior de nuestras casas, es consciente que nuestros hogares han sido el refugio en el que vamos a estar seguros. "\#YoMeQuedoEnCasa" es la manera de protegernos a nosotros mismos y a los seres más queridos y cercanos. La iniciativa de Ikea va enfocada a "amueblarnos la cabeza sin salir de casa" y ofrece de forma gratuita cuentos y pasa tiempos para los más pequeños de la casa. Una manera de mantenerlos entretenidos y para que padres y madres, puedan tener tiempo para poder desarrollar el teletrabajo o realizar tareas de la casa en familia.

GRAFICO 5. La campaña de Ikea. Fuente (Atrivity, 2020)

\section{\#YoMeQuedoEnCasa}

\author{
Ahora más que nunca, nada \\ como el hogar para amueblarnos \\ la cabeza
}




\section{LA ESTRATAGIA SOCIAL CORPORATIVA DE INDITEX. ZARA}

En 1963, este gigante de la confección, comienza su actividad en A Coruña (España), como confecciones GOA, en un modesto taller en el que realiza vestidos y batas de mujer para su distribución. En diez años, la plantilla pasó a estar formada por 500 personas. En 1975, abre la primera tienda de Zara, después de 12 años dedicados a la fabricación textil, el fundador de Inditex, Amancio Ortega, abre su primera tienda en A Coruña. (España). En el año 1976, la tienda se plantea que el cliente es su principal círculo virtuoso y lo ubica en el centro de las decisiones, lo acerca a sus preferencias y le ofrece moda a precios accesibles. En 1977, establece su sede en Arteixo (España), se construyen las fábricas GOA y Samlor en dicha localidad coruñesa, donde se encuentra la sede actualmente.

Hoy, ante la pandemia, el gigante del textil, ha donado 35 millones de unidades de protección sanitaria y 2 millones de mascarillas para centros médicos para hacer frente a la crisis del coronavirus y la falta de material.

Inditex está utilizando todo su potencial de almacenamiento y logística de Arteixo (A Coruña) para realizar envíos masivos a toda la península en tiempo récord. El material llegado desde China está embalado con el mensaje: "aunque nos separen los mares, nos une la misma luna".

GRAFICO 6. La campaña de RSC de Inditex. Zara. Fuente (Atrivity, 2020)

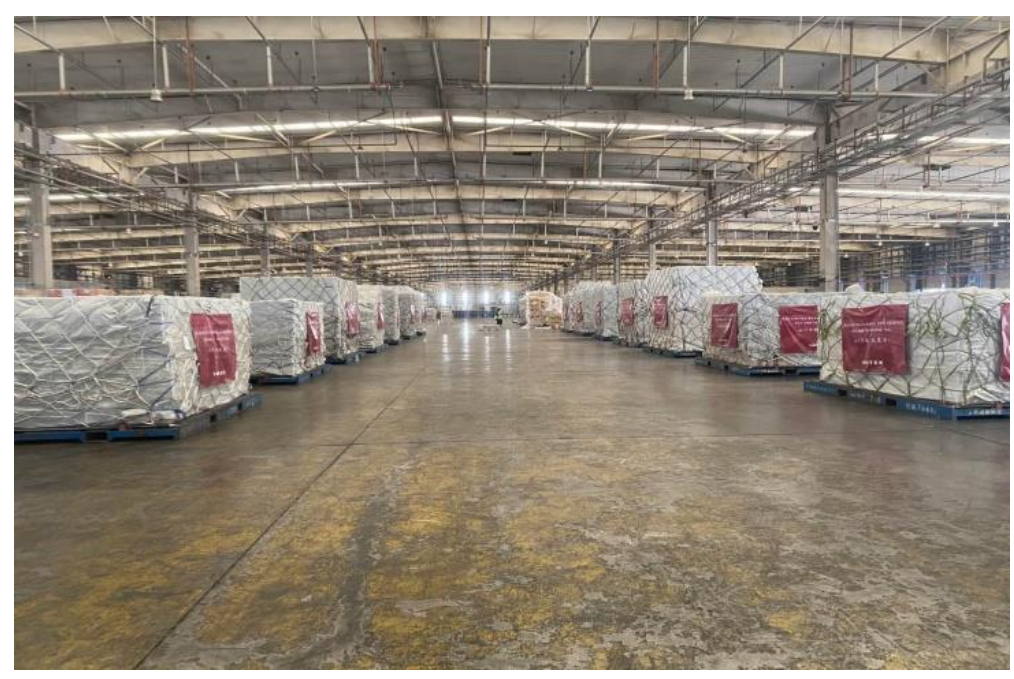

\section{ESTRATEGIAS DE DIVERSIFICACIÓN DEL NEGOCIO}

En el sector retail las marcas han optado por potenciar sus plataformas online como recurso para minimizar el impacto negativo que supone tener todas las tiendas físicas cerradas. Una tendencia que va a permanecer incluso en la época post COVID-19 en forma de omnicanal. Algunas de las mejores estrategias online han sido realizadas por Carrefour, Leroy Merlin y Decathlon. 


\section{LA ESTRATEGIA DE DIVERSIFICACIÓN DE CARREFOUR}

La cadena de supermercados Carrefour, ha potenciado en gran medida su página web, con el lanzamiento de kits de productos básicos para aquellas personas que necesiten el envío rápido de alimentos.

Se trata de lotes pre-configurados de gran consumo con entrega a domicilio entre 48 y 72 horas.

Esto representa, un nuevo giro del distribuidor francés para potenciar su ecommerce frente al incremento de la demanda online de productos de primera necesidad a raíz de la crisis del coronavirus.

\section{GRAFICO 7. La estrategia de Carrefour. Fuente (Atrivity, 2020)}

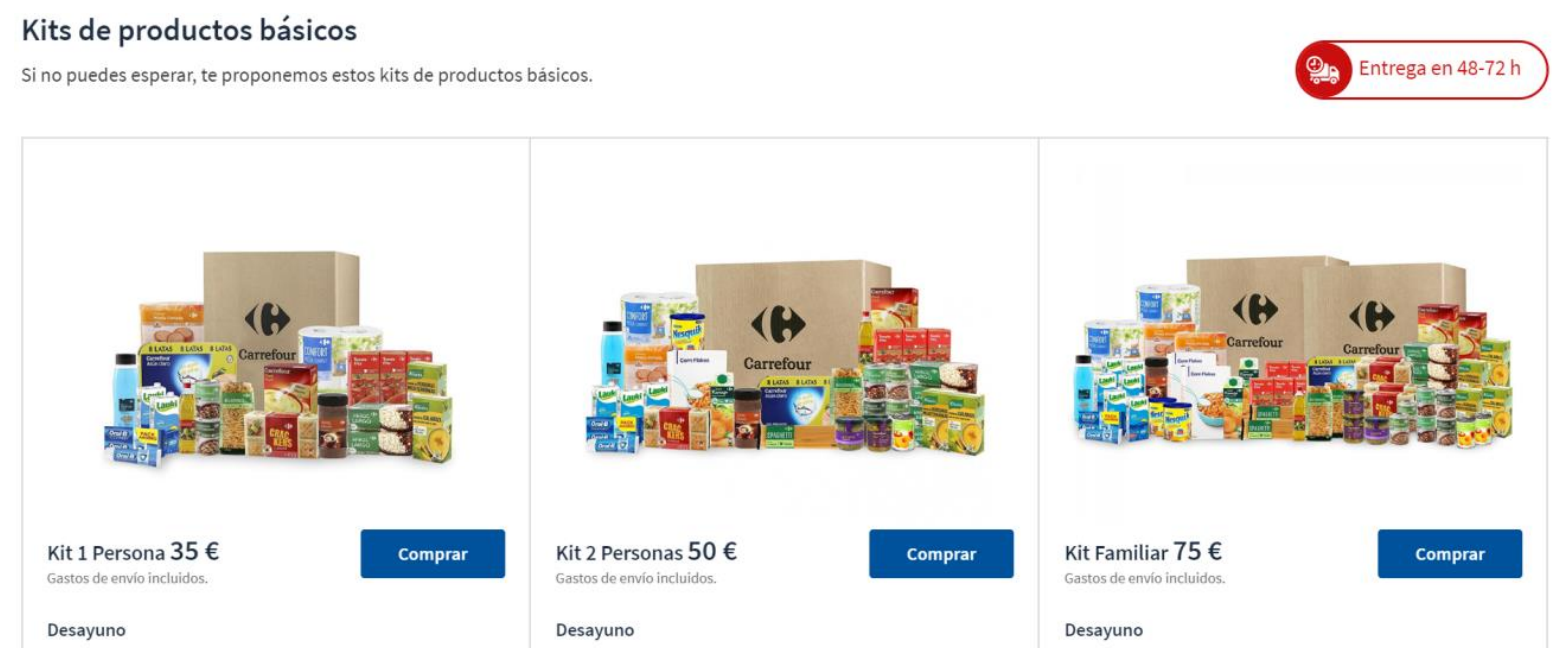

\section{LA ESTRATEGIA DE DIVERSIFICACIÓN DE LEROY MERLIN}

En 1923, los emprendedores Adolphe Leroy y Rose Merlín, abren un almacén americano de material militar recuperado, al finalizar la Primera Guerra Mundial. En 1933, animado por el éxito obtenido, el matrimonio empieza a vender en la tienda, casas prefabricadas y otros materiales de construcción. En 1947, siguiendo con su aventura, el "Stock Americain" abre almacenes suplementarios en el norte de Francia. En 1960, la marca "Stock Americain" pasa a ser "Leroy Merlin". En 1968, crea la primera tienda de auto-servicio de bricolajeferretería, en Francia, en Noeux-les-Mines. En 1973, orgullosa de su nuevo slogan, Tout pour la maison (Todo para la casa), la marca aumenta el número de referencias y puntos de venta. En 1980, la familia Mulliez, pionera en la gran distribución, adquiere la marca con sus 33 tiendas. En 1989, Leroy Merlín, se hace internacional, con la apertura de la primera tienda en España, en Leganés, al sur de Madrid. En 1996, con Polonia e Italia, la marca conquista nuevos territorios, instalándose en junio de ese año en Piaseczno, al sur de Varsovia y en octubre del mismo año en Solbiate Arno, cerca de Varese. En 1998, la 
empresa abre su primera tienda brasilera, en Interlagos, cerca de Sao Paulo. A partir del 2003, su crecimiento es superlativo, instalándose en Portugal, (Gondomar, cerca de Oporto) luego en Grecia, en el 2004, Rusia y China. A partir del 2007, abre en Gracia su primer tienda cerca del aeropuerto de Atenas. ADEO pasa a ser el nombre de la entidad que agrupa a Leroy Merlín, con otras 7 marcas de bricolaje y decoración, que tienen cada una su propia gestión.

Hoy, en escenario de planea pandemia, el gigante del bricolaje sorprende a sus clientes con una nueva sección de venta telefónica dentro de su página web. Lo que va a permitir una venta online personalizada y una mayor experiencia de compra para aquellos clientes que necesiten el producto que más se ajuste a sus necesidades.

Leroy Merlin aprovecha la situación provocada por el virus para seguir creciendo en su estrategia online y apostando por proveedores nacionales.

\section{GRÁFICO 8. La estrategia de Leroy Merlín. Fuente (Atrivity, 2020)}

\section{Haz tu compra por teléfono}

Horario venta telefónica en Semana Santa. Particulares:

jueves de $10 \mathrm{~h}$ a $20 \mathrm{~h}$. Profesionales: jueves de $7 \mathrm{~h}$ a $14 \mathrm{~h}$.

Viernes, sábado y domingo cerrado excepto en Cataluña.

Lunes cerrado en País Vasco, Navarra, Cantabria y

Cataluña. Recuerda que puedes seguir comprando en

nuestra tienda online.

\section{El caso Decathlon.}

Actualmente, millones de personas se encuentran en casa sin la posibilidad de ejercitarse como la hacían anteriormente en el gimnasio, saliendo a correr o montando en bici.

Frente a esta necesidad aparece DECATHLON COACH, una aplicación gratuita tanto para dispositivos Android e IOS, que te permite contactar con un entrenador virtual que te envía instrucciones de voz y es capaz de medir tu actividad

\section{GRÁFICO 9. EI caso DECATHON COACH. Fuente (Atrivity, 2020)}

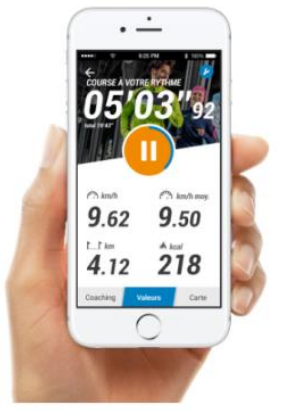

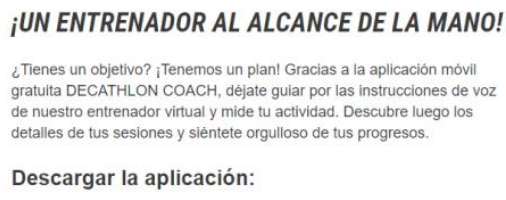

án AppStore

Más información > 


\section{ESTRATEGIAS DE FORMACIÓN Y TELETRABAJO}

Hoy muchas empresas, se encuentran en una situación complicada con la totalidad de los empleados en casa o teletrabajando, lo que puede suponer una progresiva desmotivación y desinformación por parte de su fuerza de trabajo. Por este motivo, diversas plataformas como Domestika, Microsoft Teams y Atrivity ofrecen servicios de formación para particulares como organizaciones con el objetivo de mejorar la comunicación a distancia y aprovechar el tiempo en casa para preparar a los empleados para la "vuelta a la normalidad".

\section{El caso Domestika}

La pandemia, el encierro y las restricciones han generado un inicial estado de inactividad y falta de agenda para neutralizarla. Domestika quiere fomentar el aprendizaje tanto de niños como de adultos durante estos días de confinamiento.

La plataforma online ofrece cursos gratuitos y de pago para que puedas desarrollar tu creatividad en casa. Sus contenidos van orientados al uso de todo tipo de aplicaciones e incluso a la orientación de cómo empezar con tu propio negocio online. Y comunica que esa plataforma para iniciar tu propio negocio es ¡Apto para todos los públicos!

GRÁFICO 10. El caso Doméstika. Fuente (Atrivity, 2020)

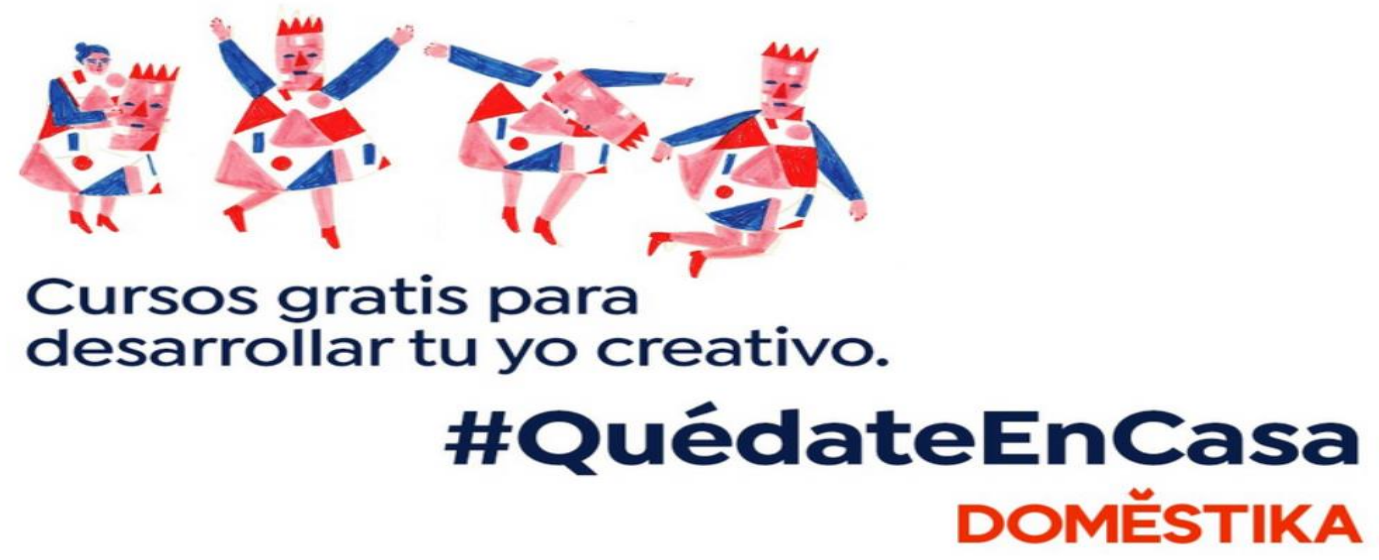

\section{LA ESTRATEGIA DE MICROSOFT TEAMS}

Microsoft ha puesto en marcha un plan de seis meses gratuitos en la versión profesional de Microsoft Teams para todos aquellos equipos que se encuentran teletrabajando. Una herramienta que permite a los empleados realizar chat, llamadas, reuniones y colaborar entre sí.

Una iniciativa que tiene la intención de mejorar la experiencia comunicativa a distancia entre compañeros de trabajo y que va acompañada de la consigna "trabaja remotamente sin sentir la distancia". 


\section{GRÁFICO 11. La estrategia de Microsoft Teams. Fuente (Atrivity)}

Microsoft Teams

Work remotely without feeling remote.

\section{Sign in}

Get your school signed up for free >

Just need video calling for personal use? Click here >
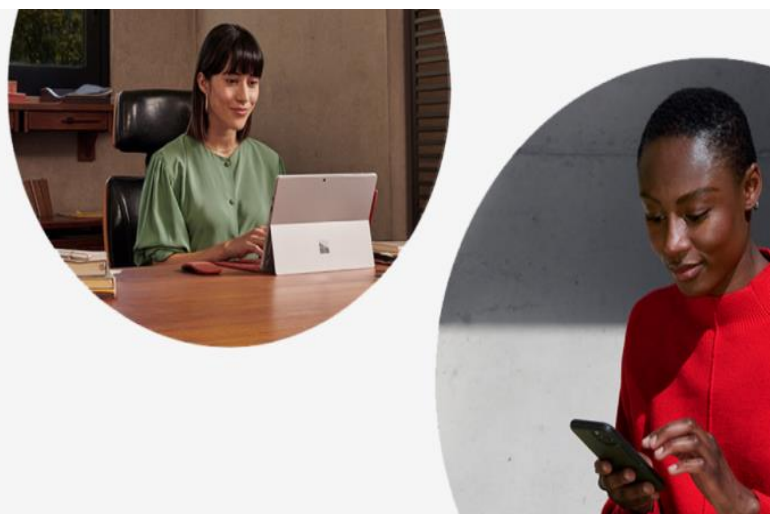

\section{LA ESTRATEGIA DE ATRIVITY}

Atrivity, es una aplicación móvil gamificada orientada para todos aquellos managers que necesiten ofrecer formaciones y comunicados empresariales relevantes para tus equipos. Una de las principales características de Atrivity es que puede contribuir a potencial el acceso y el uso de otras plataformas LMS.

De esta manera podrás aprovechar al máximo este "pit stop" provocado por la pandemia del coronavirus manteniendo a tus empleados motivados, capacitados y preparados para el "retorno a la normalidad".

Soluciones cómo Atrivity y su nueva sección de Feed, permiten comunicar de forma instantánea y personalizada cualquier información o novedad corporativa. La ventaja competitiva que ofrece a los usuarios, es la posibilidad de asociar el contenido formativo con dinámicas de juego que aseguran un aprendizaje exitoso.

Además, podrás evaluar y medir los resultados de los empleados para poder reforzar posteriormente las lagunas del conocimiento y minimizando la curva del olvido.

\section{GRÁFICO 12. La estrategia de Atrivity. Fuente (Atrivity, 2020)}
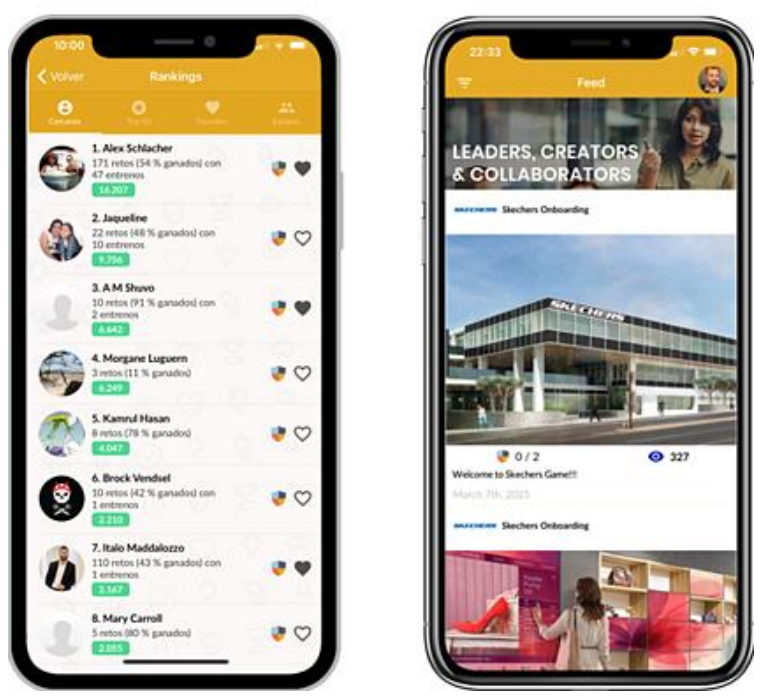


\section{LAS ESTRATEGIAS PUSH DE LA FÁBRICA AL HOGAR. EL CASO HAVANNA}

En abril del 2021, Havanna reveló la fórmula de sus alfajores desde su cuenta de Instagram y le dice a sus clientes ahora los podés hacer en casa.

Transmite tres conceptos fuertes: la generosidad de compartir su fórmula, su seguridad sobre su calidad de origen, que hace que siempre el alfajor sea mejor comprado en los canales de la marca, la empatía con el hogar, en el que las actividades culinarias, representaron en tiempo de pandemia, una de las principales actividades aglutinadoras de valor.

GRÁFICO 13. El caso Havanna. Fuente (Amigo, 2021)

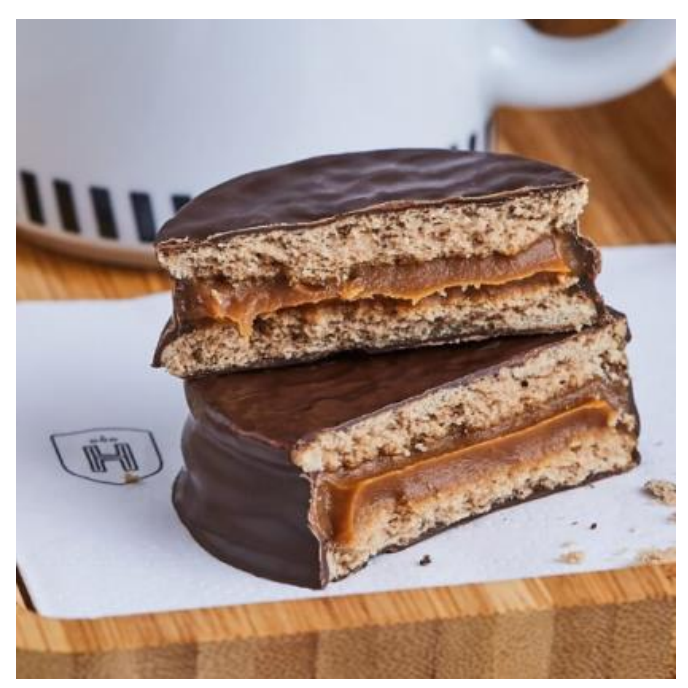

\section{Havanna reveló la receta de \\ sus alfajores y podes hacerla en casa}

Y dan el toque secreto para que la textura quede igual a la original.

Hay sabores que son únicos y los tradicionales alfajores Havanna son dueños de uno de los mejores de la Argentina.

Desde su cuenta oficial de Instagram la marca publicó recetas de sus productos típicos para que el aislamiento social, preventivo y obligatorio en el marco del coronavirus no nos prive de este enorme placer. Abril 2021

\section{LOS CIRCULOS VIRTUOSOS}

¿Qué pudimos observar en todos estos casos? Cuál fue la acción más trascendente en todas las empresas citadas? 
Hubo un verbo constante. Las empresas aplicaron el dar... Quizás se olvidaron de la rentabilidad, pero ganaron en confiabilidad, en credibilidad, su imagen corporativa, finalmente creció y es ese recurso el encargado de devolver la rentabilidad.

Asistimos a la representación vívida de lo que es un círculo virtuoso Un círculo, tiene un punto que lo inicia. ¿Quién motiva a un círculo virtuoso? ¿La sociedad? ¿Un hombre? ¿Una mujer desprotegida? ¿La desnutrición? ¿La inseguridad? ¿Un jubilado enfermo y sin ingresos? ¿La libertad? ¿Los niños? ¿Los adolescentes con ilusiones? ¿Los adolescentes desmotivados? ¿Los targets apriorísticos? ¿Las sociedades que Giddens llama "sociedades de encierro? Amigo (2012; 2017).

Según Bernardo Klisberg en su obra "Más ética, más desarrollo" (41) expresa que Noruega es uno de los líderes mundiales en transparencia. Y me pregunto si la transparencia es un condimento positivo o negativo para la vida, para la vida social, para la gestión de negocios.

¿Sería posible la transparencia en la Argentina? ¿Cómo responderían las distintas organizaciones ante la transparencia?

Los círculos virtuosos son aquellos que tienen ciertas características simultáneas:

- Objetivos orientados al mercado

- Objetivos compartidos

- Compromiso compartido

- Red de relaciones basada no en la estructura jerárquica sino en el nivel de prescindencia respecto de su implicancia en la estrategia corporativa (Amigo, 2012, p.41)

\section{LA COPARTICIPACIÓN}

Se deberá negociar la coparticipación. No es un proceso fácil, a veces no se logra linealmente. Implica la actitud y la aptitud inteligente del pensamiento bilateral, con consciencia de mejora. $Y$ en tiempos de pandemia, en la que todos los roles perdieron y siguen perdiendo, la clave de la coparticipación es la empatía por la realidad del otro. Y debería ser un ejercicio, que comienza desde el inicio del día.

La coparticipación necesita de responsables, que la ejecuten, la formulen, la implemente y la inspiren. Sólo un líder puede gestar la coparticipación, ya que el líder es aquel que hace que todos jalen en la misma dirección y desde la ejemplaridad genera el proceso de influencia necesario y suficiente para la compatibilización entre pares, entre actores de un sector industrial, entre proveedores y clientes, entre el compromiso por la estrategia de la empresa o copiar la estrategia de los competidores, entre funcionarios de distintas áreas de la empresa, entre el frente externo y el frente interno. 
El proceso de la compatibilización de los objetivos para lograr objetivos compartidos, requiere de una metodológica política de negociación, en la que no perderemos la perspectiva en la función básica proporcionada al mercado y en la posibilidad de la trascendencia.

Sólo los objetivos compartidos se cumplen sin obstáculos internos en su camino.

Por eso, deberemos asignarle al tiempo de la coparticipación, toda la asistencia metodológica para una vez fijados, los mismos sirvan de inspiradoración para la formulación de las políticas comerciales.

Procter and Gamble, reconoce que está hecha de personas cuyos aportes se combinan para formular el desempeño del todo. "Ninguna compañía puede crecer y llegar a ser grande sólo a base de las habilidades de sus propietarios. P\&G reconoció desde muy pronto, antes que muchas otras compañías grandes, la noción de que los intereses del empleador y de sus empleados son inseparables y que las personas merecen un tratamiento digno (Dyer y otros, 2005)

Este principio es la esencia del compromiso compartido, el que deberá recorrer la siguiente secuencia:

1- Reconocer una secuencia:

2- Compromiso imprescindible

3- Reconocimiento de las personas involucradas, independientemente de su nivel jerárquico.

4- Comunión de objetivos.

5- El reconocimiento avala y apalanca la iniciativa privada, convirtiendo a las personas en enterpreneur de su propia responsabilidad y de su resultado consecuente.

6- Reconocer que necesito de las personas

7- Sobre la base de la ejemplaridad, establecer una mirada compartida hacia el mercado en donde todos los sectores, estarán mirando hacia esa dirección y el directivo estará a disposición de todos y cada uno de los niveles responsables de transmitir valor y entregarlo

\section{CONCLUSIONES}

- Siempre las crisis esconden grandes oportunidades, este decir se torna difícil de llevarlo a la práctica, cuando acaece realmente, es decir cuando la adversidad se convierte en la realidad de la empresa. Sin embargo, es el único camino para neutralizar la adversidad y así ser capaz de sacar el ser más generoso que llevamos dentro. 
- Las distintas campañas lanzadas por el conjunto de organizaciones demuestran que el COVID-19 ha hecho aparecer la mejor versión de nosotros, ayudando en gran medida a hacer más llevaderos los días de cuarentena.

- Promover e incentivar el conjunto de los valores corporativos, hoy constituyen el único medio para impulsar las relaciones entre los clientes internos y van a cobrar una gran importancia en el momento de fidelizar clientes y fortalecer los vínculos que nos unen.

- La clave es fortalecer los vínculos entre clientes internos y clientes externos, interpretar esos vínculos para poder desarrollar finalmente las ventajas competitivas sostenibles más difíciles de imitar y repetir por terceros.

- Los círculos virtuosos, salieron a la luz, mostrando lo mejor de las empresas para brindarlo al mercado.

- El compromiso que implican, es que esos círculos virtuosos se han potenciado, se han instalado en la sociedad, la sociedad los requiere y constituyen el mejor ancla en los vínculos interpersonales y entre empresariales, ya no podrás convertirse en recuerdos virtuosos, son la realidad de la sociedad post pandemia.

\section{REFERENCIAS BIBLIOGRÁFICAS:}

Aaker, David (1994). Gestión del Valor de la Marca. Díaz de Santos. Madrid.

Amigo, Adriana (2009). Negocios con Valor, $1^{\circ}$ Edición. Fundación Ross, Argentina.

Amigo, Adriana (2012). Negocios con Valor. $2^{\circ}$ Edición. Fundación Ross, Argentina.

Amigo, Adriana (2017). Negocios con Valor. Edición Europea. Editorial Académica Española. España. Madrid.

Buckley, John V. (2000). Cómo crecer con ventaja competitiva. El valor real de la Tecnología. Mc Graw Hill. Colombia.

Carrión Marotto, Juan (2007). Estrategia, de la visión a la acción. Alfaomega, ESIC, España. Dyer, Davis; Dalzell, Frederick; Olegario, Roxana (2005). Procter \& Gamble (Rising Tide) 165 años construyendo una marca. Grupo Editorial Norma. Bogotá. Colombia.

Gibson, Rowan y otros (1997). Repensando el futuro, Grupo Editorial Norma, Bogotá. Colombia.

Hamel, Gary; Prahalad, G. K. (1999). Compitiendo por el futuro, Editorial Ariel, Sociedad Económica, 2ํㅜ reimpresión, Barcelona, España.

Kliksberg, Bernardo (2004). Más ética, más desarrollo. Temas, $1^{\circ}$ Edición. Buenos Aires. Argentina.

Simon, Herbert (1980). El comportamiento Administrativo. Un estudio de los procesos de decisión en las organizaciones administrativas. Aguilar. Buenos Aires. Argentina. 


\section{FUENTES:}

blog.atrivity.com/es/estrategia-comunicación-creatividad-covid19.Última actualización 16 de abril de 2020. Martínez, Roger.

https://www2.deloitte.com/ar/es/pages/about-deloitte/articles/atendiendo-impacto-financierocovid19.html

https://elpais.com/economia/2020/08/20/nuevos tiempos/1597919911 259535.html 